\title{
THE SECRETARY PROBLEM OF MINIMIZING THE EXPECTED RANK: A SIMPLE SUBOPTIMAL APPROACH WITH GENERALIZATIONS
}

\author{
ABBA M. KRIEGER, ${ }^{*}$ University of Pennsylvania \\ ESTER SAMUEL-CAHN, ${ }^{* *}$ The Hebrew University of Jerusalem
}

\begin{abstract}
The secretary problem for selecting one item so as to minimize its expected rank, based on observing the relative ranks only, is revisited. A simple suboptimal rule, which performs almost as well as the optimal rule, is given. The rule stops with the smallest $i$ such that $R_{i} \leq i c /(n+1-i)$ for a given constant $c$, where $R_{i}$ is the relative rank of the $i$ th observation and $n$ is the total number of items. This rule has added flexibility. A curtailed version thereof can be used to select an item with a given probability $\mathrm{P}, \mathrm{P}<1$. The rule can be used to select two or more items. The problem of selecting a fixed percentage, $\alpha, 0<\alpha<1$, of $n$, is also treated. Numerical results are included to illustrate the findings.
\end{abstract}

Keywords: Secretary problem; group selection rules; suboptimal rule; near optimal rule; absolute rank; relative rank

2000 Mathematics Subject Classification: Primary 62L99

Secondary 62F07; 60F $15 ; 60 \mathrm{~F} 15$

\section{Introduction}

Consider a group of $n$ items that arrive sequentially and are rankable without ties. The number of items, $n$, is known. A rank of 1 denotes the best item, a rank of 2 denotes the second best item, etc. It is assumed that all $n$ ! permutations of the ranks $\{1, \ldots, n\}$ are equally likely.

The problem is to establish a rule that decides whether item $i$ is to be chosen based on its relative rank, $R_{i}$, i.e. its rank among the first $i$ items. Just as in the classical secretary problem, once a decision is made on an item, there is no recourse.

The performance of the rule depends on the absolute rank of item $i, A_{i}^{n}$, i.e. its rank among all $n$ items, if it is chosen. In the classical secretary problem the objective is to choose a single item by means of an optimal stopping rule $\tau_{n}$ that maximizes $\mathrm{P}\left(A_{\tau_{n}}^{n}=1\right)$.

Chow et al. [2] considered the problem of finding an optimal stopping rule $\tau_{n}$ for choosing one item that minimizes the expected value of the absolute rank of the item selected. They showed that the optimal stopping rule satisfies

$$
\lim _{n \rightarrow \infty} \mathrm{E}\left(A_{\tau_{n}}^{n}\right)=\prod_{j=1}^{\infty}\left(1+\frac{2}{j}\right)^{1 /(j+1)}=3.869 .
$$

\footnotetext{
Received 16 January 2009; revision received 9 August 2009.

* Postal address: Department of Statistics, The Wharton School, University of Pennsylvania, Philadelphia, PA 191046340, USA. Email address: krieger@wharton.upenn.edu

Supported by funds from the Marcy Bogen Chair of Statistics at the Hebrew University of Jerusalem.

** Postal address: Department of Statistics (and Center for Rationality), The Hebrew University of Jerusalem, Jerusalem, 91905, Israel. Email address: scahn@mscc.huji.ac.il

Supported by the Israel Science Foundation Grant 467/04.
} 
This is an astonishing result since it shows that, for example, from one million items we can sequentially select an item with expected rank less than 4 . Note that, since one item must be chosen, if no item has been chosen among the $n-k$ first items, where $k$ is small relative to $n$, we must choose an item among the last $k$ items. Since the last few items have expected absolute rank $(n+1) / 2$, the probability of this happening must be at most $O(1 / n)$, for the expected rank of the chosen item not to tend to infinity. The paper by Chow et al. [2] is heavily cited and has spawned many variations.

The optimal stopping rule in Chow et al. for keeping one item can in principle be determined by dynamic programming and so in practice is hard to implement for large $n$. The asymptotic form of the rule is to pass on the first $\gamma_{0} n$ items; choose the first item with $R_{i}=1$ after $i>\gamma_{0} n$ and up to $\gamma_{1} n$, choose the first item with $R_{i} \leq 2$ after $\gamma_{1} n$ (if none has been chosen before) and up to $\gamma_{2} n$, and so on. The values of the $\gamma_{i}$ s can be computed asymptotically. For example, from Ferguson [4, p. 26], $\gamma_{0}=0.2584, \gamma_{1}=0.4476$, and $\gamma_{2}=0.5640$.

We consider finding simple stopping rules that perform well in minimizing the sum of the expected value of the absolute ranks of the items selected, when one or more items are desired. In Section 2 we pose a class of rules which are easy to implement, and specifically a rule that achieves asymptotic expected absolute rank of 3.928, nearly the same as the optimal rule. The rules that are posed have added flexibility. They allow for stopping rules that select an item only with high probability of stopping. We can find rules that do substantially better than the optimal expected absolute rank of 3.89 by slightly relaxing the 'must' select an item only condition. For example, when the probability of stopping is at least $95 \%$, the asymptotic expected absolute rank is 2.987 . We can find rules that do substantially better than (1.1) while slightly relaxing the 'must select an item' only. Yeo and Yeo [7] also considered stopping rules for secretary problems which do not necessarily stop with probability 1 , and included some asymptotic evaluations. Our approach is different from theirs in that we are considering simple suboptimal rules that perform well.

In Section 3 we consider the problem of selecting a fixed number, $r$, of items, $r>1$. The optimal stopping rule can again be determined by dynamic programming. The simple rule we establish for $r=1$ can be adapted to this problem as well, again producing nearly optimal results.

The above rules fix the number of items selected. Other rules select a random number of items satisfying a condition on the average number of items retained; for example, the average number that is retained is a given percentage of $n$. The problem of sequentially selecting a group of items on the basis of their relative ranks has recently been described in Krieger et al. [5]. They considered a class of rules termed 'percentage rules' for $0<p \leq 1$, with the property that they retain on average an order of $n^{p}$ items, most of which are good. Simple rules that perform asymptotically optimally in terms of the sum of the absolute ranks and retain (approximately) a fixed given fraction $\alpha$ are described in Section 4. For the rules in [5], as well as for the rules in Section 4, no prior knowledge of $n$ is needed. In Section 5 we supply tables illustrating the results obtained in the earlier sections.

\section{Retaining one item}

The optimal rule to retain one item that minimizes the expected value of the absolute rank can be found using dynamic programming for any $n$. This rule can be described by a sequence of integers $r_{n}(j), j=0, \ldots, n$, with $1 \leq r_{n}(0) \leq r_{n}(1) \leq \cdots \leq r_{n}(n)=n+1$. Let $i$ denote the index of the item. If $i<r_{n}(0)$ then the item is never accepted. In general, item $i$ is chosen when its relative rank $R_{i} \leq j$ and $i<r_{n}(j)$, provided that no item has been retained earlier. 
In this section we consider simple rules that are easy to implement (i.e. with values of $r_{n}(j)$ that are readily available). To this end, let $c \geq 1$ be a fixed constant, and let the stopping rule be

$$
t_{n}(c)=\inf \left\{i: R_{i} \leq \frac{i c}{n+1-i}\right\} .
$$

Note that the stopping rule $t_{n}(c)$ satisfies $\mathrm{P}\left(t_{n}(c) \leq n\right)=1$.

We shall suppress the dependence on $c$ in some of the following notation, where we consider $c$ as fixed. Let $a_{n}(k)$ be the smallest integer greater than or equal to $(n+1) k /(c+k)$, i.e.

$$
a_{n}(k)=\left\lceil\frac{(n+1) k}{(c+k)}\right\rceil, \quad k=1, \ldots, n .
$$

Note that, for small values of $k$ (as compared to $n$ ), the values of $a_{n}(k)$ will be distinct, but, for large values of $k$, some consecutive $a_{n}(k)$ may coincide. Clearly, $t_{n}(c)$ in (2.1) can be written as

$$
t_{n}(c)=\inf \left\{i: R_{i}=k \text { for some } i \geq a_{n}(k), k=1, \ldots, n\right\} .
$$

One of our main interests will be in a 'curtailed' version of (2.2), which stops with probability less than 1 . Let $k_{s}$ be a fixed integer with $k_{s}<n$. Define

$$
t_{k_{s}, n}(c)=\inf \left\{i: R_{i}=k \text { for some } i \geq a_{n}(k), k=1, \ldots, k_{s}\right\} .
$$

Clearly, $t_{k_{s}, n}(c)$ will stop with probability less than 1 . For fixed $c$ and $k_{s}$, we are interested in finding the limiting probability of stopping, and the expected absolute rank upon stopping, as $n \rightarrow \infty$. This is used later to determine $c$ and $k_{s}$ to attain a desired probability $\mathrm{P}$ of stopping.

Let $b_{n}(k)=a_{n}(k+1)-1$ for $k=1, \ldots, k_{s}-1$, and let $b_{n}\left(k_{s}\right)=n$. Assume that $n$ is large, so that $b_{n}(k)>a_{n}(k)$ for $k=1, \ldots, k_{s}$. Let $Q_{n}(k)=\left\{R_{i}>j\right.$ for all $i \leq b_{n}(j)$ and all $j=1, \ldots, k\}$. Thus,

$$
\mathrm{P}\left(Q_{n}(k)\right)=\mathrm{P}\left(t_{k_{s}, n}(c)>b_{n}(k)\right) \text { for } k=1, \ldots, k_{s}-1
$$

(where not stopping corresponds to $t=\infty$ ) and

$$
\mathrm{P}_{n}\left(k_{s}, c\right)=\mathrm{P}\left(t_{k_{s}, n}(c)<\infty\right)=1-\mathrm{P}\left(Q_{n}\left(k_{s}\right)\right)
$$

where $Q_{n}\left(k_{s}\right)$ is defined by the above expression and $Q_{n}(0)$ is the entire space.

Lemma 2.1. For $k \leq k_{s}$, let

$$
f(k):=\lim _{n \rightarrow \infty} \mathrm{P}\left(Q_{n}(k) \mid Q_{n}(k-1)\right)=\lim _{n \rightarrow \infty} \prod_{j=a_{n}(k)}^{b_{n}(k)}\left(1-\frac{k}{j}\right) .
$$

Then, for $k=1, \ldots, k_{s}-1$,

$$
f(k)=\left(\frac{k(k+c+1)}{(k+c)(k+1)}\right)^{k}
$$

and

$$
f\left(k_{s}\right)=\left(\frac{k_{s}}{k_{s}+c}\right)^{k_{s}}
$$


Proof. To see (2.6), note that

$$
\begin{aligned}
\lim _{n \rightarrow \infty}\left(\log \prod_{j=a_{n}(k)}^{b_{n}(k)}\left(1-\frac{k}{j}\right)\right) & =\lim _{n \rightarrow \infty} \sum_{j=a_{n}(k)}^{b_{n}(k)} \log \left(1-\frac{k}{j}\right) \\
& =\lim _{n \rightarrow \infty}\left(-k \sum_{j=a_{n}(k)}^{b_{n}(k)} \frac{1}{j}\right) \\
& =-k \lim _{n \rightarrow \infty}\left[\log b_{n}(k)-\log a_{n}(k)\right] \\
& =\lim _{n \rightarrow \infty} \log \left(\frac{a_{n}(k)}{b_{n}(k)}\right)^{k} \\
& =\log \left(\frac{k(k+c+1)}{(k+c)(k+1)}\right)^{k} .
\end{aligned}
$$

Equation (2.7) follows similarly.

Proposition 2.1. We have

$$
\begin{aligned}
\mathrm{P}\left(k_{s}, c\right) & :=\lim _{n \rightarrow \infty} \mathrm{P}_{n}\left(k_{s}, c\right) \\
& =\lim _{n \rightarrow \infty} \mathrm{P}\left(t_{k_{s}, n}(c)<\infty\right) \\
& =1-\prod_{k=1}^{k_{s}} f(k) \\
& =1-\frac{k_{s} !}{\prod_{k=1}^{k_{s}}(k+c)} .
\end{aligned}
$$

Proof. The proof follows from (2.4), (2.5), and Lemma 2.1.

To compute the expected absolute rank upon stopping, note that

$$
\mathrm{E}\left(A_{i}^{n} \mid R_{i}\right)=\frac{n+1}{i+1} R_{i}
$$

where $A_{i}^{n}$ is the absolute rank, among $n$, of the $i$ th observation. It follows that $\mathrm{E}\left(A_{i}^{n} \mid R_{i} \leq\right.$ $k)=(n+1)(k+1) / 2(i+1)$. We are interested in computing $\mathrm{E}\left(A_{t_{k_{s}, n(c)}}^{n} \mid t_{k_{s}, n}(c)<\infty\right):=$ $\mathrm{E}\left(n, k_{s}, c\right)$, as well as its limiting value, to be denoted by $\mathrm{E}\left(k_{s}, c\right)$. Let $V_{n}(k)=\mathrm{P}\left(t_{k_{s}, n}(c)>\right.$ $\left.b_{n}(k-1)\right)$ for $k=2, \ldots, k_{s}$ and $V_{n}(1)=1$. Note that, for $k=2, \ldots, k_{s}$, from (2.6),

$$
\lim _{n \rightarrow \infty} V_{n}(k):=V(k)=\prod_{j=1}^{k-1} f(j)=\frac{(k-1) !}{\prod_{j=1}^{k-1}(j+c)}\left(\frac{k+c}{k}\right)^{k-1} .
$$

We now find the conditional (on an item being retained) expected absolute rank, $\mathrm{E}\left(n, k_{s}, c\right)$, and its limit as $n \rightarrow \infty, \mathrm{E}\left(k_{s}, c\right)$ in (2.11), below.

Theorem 2.1. We have

$$
\mathrm{E}\left(k_{s}, c\right)=\frac{\sum_{j=1}^{k_{s}} \alpha_{j-1} \beta_{j}-k_{s} \alpha_{k_{s}}}{2 \mathrm{P}\left(k_{s}, c\right)}
$$


and when $c>1$,

$$
\lim _{k_{s} \rightarrow \infty} \mathrm{E}\left(k_{s}, c\right):=\mathrm{E}(c)=\frac{1}{2} \sum_{j=1}^{\infty} \alpha_{j-1} \beta_{j}
$$

where

$$
\alpha_{m}=\frac{m !}{\prod_{j=1}^{m}(j+c)}=\left[\prod_{j=1}^{m}\left(1+\frac{c}{j}\right)\right]^{-1}
$$

and

$$
\beta_{m}=\left(\frac{m+c}{m}\right)^{m}=\left(1+\frac{c}{m}\right)^{m}
$$

with $\alpha_{0}=1$.

Proof. We shall compute the expected value $\mathrm{E}\left(n, k_{s}, c\right)$ by considering in which of the disjoint intervals $\left[a_{n}(k), b_{n}(k)\right]$ for $k=1, \ldots, k_{s}$, and at which value $i$ inside that interval, the rules stop. It is easily seen that

$$
\mathrm{E}\left(n, k_{s}, c\right)=\sum_{k=1}^{k_{s}} V_{n}(k)\left(\sum_{i=a_{n}(k)}^{b_{n}(k)}\left[\prod_{m=a_{n}(k)}^{i-1}\left(1-\frac{k}{m}\right)\right] \frac{k}{i} \frac{(n+1)(k+1)}{2(i+1)}\right) / \mathrm{P}_{n}\left(k_{s}, c\right) .
$$

To evaluate the limit, note that, for $k \leq k_{s}$, the value of $i$ will be a fraction of $n$. Thus, denote $i / n=x$. The term

$$
\prod_{m=a_{n}(k)}^{i-1}\left(1-\frac{k}{m}\right)=\frac{(i-1-k) \cdots\left(a_{n}(k)-k\right)}{(i-1) \cdots a_{n}(k)} .
$$

Dividing the numerator and denominator by $n$ and letting $n \rightarrow \infty$ yields $(k /(k+c))^{k}$ in the numerator and $x^{k}$ in the denominator. Then it follows that, for $k<k_{s}$,

$$
\begin{aligned}
\lim _{n \rightarrow \infty} & \sum_{i=a_{n}(k)}^{b_{n}(k)}\left[\prod_{m=a_{n}(k)}^{i-1}\left(1-\frac{k}{m}\right)\right] \frac{n+1}{i(i+1)} \\
& =\left[\frac{k}{k+c}\right]^{k} \int_{k /(k+c)}^{(k+1) /(k+1+c)} x^{-(k+2)} \mathrm{d} x \\
& =\left[\frac{k}{k+c}\right]^{k}\left[\left(\frac{k+c}{k}\right)^{k+1}-\left(\frac{k+1+c}{k+1}\right)^{k+1}\right] /(k+1),
\end{aligned}
$$

and, similarly, the corresponding limit for $k_{S}$ is

$$
\left[\frac{k_{s}}{k_{s}+c}\right]^{k_{s}} \int_{k_{s} /\left(k_{s}+c\right)}^{1} x^{-\left(k_{s}+2\right)} \mathrm{d} x=\left[\frac{k_{s}}{k_{s}+c}\right]^{k_{s}}\left[\left(\frac{k_{s}+c}{k_{s}}\right)^{k_{s}+1}-1\right] /\left(k_{s}+1\right) .
$$

Taking limits of (2.13) by substituting the limits in (2.10), (2.14), and (2.15) and simplifying somewhat, we obtain

$$
\begin{array}{r}
\mathrm{E}\left(k_{s}, c\right)=\left(\sum_{k=1}^{k_{s}-1} \frac{k ! k}{\prod_{j=1}^{k}(j+c)}\left[\left(\frac{k+c}{k}\right)^{k+1}-\left(\frac{k+1+c}{k+1}\right)^{k+1}\right]\right. \\
\left.+\frac{k_{s} ! k_{s}}{\prod_{j=1}^{k_{s}}(j+c)}\left[\left(\frac{k_{s}+c}{k_{s}}\right)^{k_{s}+1}-1\right]\right) / 2 \mathrm{P}\left(k_{s}, c\right),
\end{array}
$$


where $\mathrm{P}\left(k_{s}, c\right)$ is given in (2.8). We can simplify (2.16) somewhat by opening the square brackets and canceling to obtain

$$
\mathrm{E}\left(k_{s}, c\right)=\left(\sum_{k=1}^{k_{s}} \frac{(k-1) !}{\prod_{j=1}^{k-1}(j+c)}\left(\frac{k+c}{k}\right)^{k}-\frac{k_{s} ! k_{s}}{\prod_{j=1}^{k_{s}}(j+c)}\right) / 2 \mathrm{P}\left(k_{s}, c\right) .
$$

Result (2.11) follows from the definitions of $\alpha_{m}$ and $\beta_{m}$.

Note that the negative term in (2.11) for $c=1$ equals $k_{s} /\left(k_{s}+1\right)<1$ and is a decreasing function of $c$. We are interested in $\mathrm{E}(c)=\lim _{k_{s} \rightarrow \infty} \mathrm{E}\left(k_{s}, c\right)$. Clearly, from (2.8), $\lim _{k_{s} \rightarrow \infty} \mathrm{P}\left(k_{s}, c\right)=1$. Since $\alpha_{m} m^{c} \rightarrow$ constant as $m \rightarrow \infty$ and $\beta_{m} \rightarrow \mathrm{e}^{c}$ as $m \rightarrow \infty$, it follows that, for $c>1, \lim _{k_{s} \rightarrow \infty} k_{s} \alpha_{k_{s}} \rightarrow 0$, and we have (2.12).

When $0<c \leq 1$, however, the sum in (2.12) is infinite.

An important issue is to show that the rule in (2.2) which is not curtailed behaves like the limit of the curtailed rule with $k_{s} \rightarrow \infty$ as given in (2.12) when $n \rightarrow \infty$. It is sufficient to consider the curtailed rule with $n=k_{s}$ as $n \rightarrow \infty$.

Theorem 2.2. Let $t_{n}(c)$ be as in (2.1) with $c>1$. Then

$$
\lim _{n \rightarrow \infty} \mathrm{E} A_{t_{n}(c)}^{n}=\mathrm{E}(c),
$$

where $\mathrm{E}(c)$ is given in (2.12).

Proof. In order to show that the convergence of $\mathrm{E}\left(n, k_{s}, c\right)$ is uniform in $k_{s}$ as $n \rightarrow \infty$, we need to consider the remainder term from (2.13) (for $n>k_{0}$ large),

$$
R_{n}\left(k_{0}\right)=\sum_{k=k_{0}}^{k_{s}} V_{n}(k)\left(\sum_{i=a_{n}(k)}^{b_{n}(k)}\left[\prod_{m=a_{n}(k)}^{i-1}\left(1-\frac{k}{m}\right)\right] \frac{k}{i} \frac{(n+1)(k+1)}{2(i+1)}\right) .
$$

We want to show that $R_{n}\left(k_{0}\right)$ can be made arbitrarily small by choosing $k_{0}$ to be sufficiently large. We consider (2.17) in three parts.

1. For $V_{n}(k)$, we have

$$
V_{n}(k)=\prod_{j=1}^{k-1} \prod_{m=a_{n}(j)}^{b_{n}(j)}\left(1-\frac{j}{m}\right)<\prod_{j=1}^{k-1}\left(1-\frac{j}{b_{n}(j)}\right)^{b_{n}(j)-a_{n}(j)} .
$$

But $b_{n}(j)-a_{n}(j) \geq(n+1) c /(c+j+1)(c+j)-1$. Hence, with $k$ and $n$ fixed for positive finite constants $A, B$, and $D$ (used later),

$$
\begin{aligned}
V_{n}(k) & <\exp \left[-\sum_{j=1}^{k-1} \frac{j(c+j+1)}{(n+1)(j+1)}\left(\frac{(n+1) c}{(c+j+1)(c+j)}-1\right)\right] \\
& <A \exp \left[-c \sum_{j=1}^{k-1} \frac{j}{(j+1)(c+j)}\right] \\
& =A \exp \left[-c\left(\sum_{j=1}^{k-1} \frac{1}{c+j}-\sum_{j=1}^{k-1} \frac{1}{(j+1)(c+j)}\right)\right] \\
& <B \exp [-c \log (c+k-1)] \\
& =B(c+k-1)^{-c} .
\end{aligned}
$$


2. Next we consider the middle term in (2.17), which refers to the probability of obtaining a value in $\left[a_{n}(k), b_{n}(k)\right]$ which would force us to stop for a given $k$. Note that, for $k_{s}$, we can bound this probability trivially by 1 . For $k<k_{s}$, an upper bound is $c /(k+1)$. The upper bound follows from

$$
\begin{aligned}
\sum_{i=a_{n}(k)}^{b_{n}(k)} & \prod_{m=a_{n}(k)}^{i-1}\left(1-\frac{k}{m}\right) \frac{k}{i} \\
& =1-\prod_{m=a_{n}(k)}^{b_{n}(k)}\left(1-\frac{k}{m}\right) \\
\leq & 1-\left(1-\frac{k(c+k)}{(n+1) k}\right)^{(n+1) c /(c+k+1)(c+k)} \\
\leq & \left(\frac{k(c+k)}{(n+1) k}\right)\left(\frac{(n+1) c}{(c+k)(c+k+1)}\right) \\
= & \frac{c}{c+k+1} \\
\leq & \frac{c}{k+1} .
\end{aligned}
$$

3. Finally, we bound the expected absolute rank, for $i$ in $\left[a_{n}(k), b_{n}(k)\right]$, by

$$
\frac{(n+1)(k+1)}{2(i+1)} \leq \frac{(n+1)(k+1)(c+k)}{2(n+1) k}<(c+1)(k+1) .
$$

Substituting (2.18), (2.19), and (2.20) into (2.17) yields

$$
R_{n}\left(k_{0}\right) \leq D \sum_{k=k_{0}}^{k_{s}-1}(c+k-1)^{-c}+(c+1)\left(k_{s}+1\right) B\left(c+k_{s}-1\right)^{-c} .
$$

It is clear that, for $c>1$, the sum in (2.21) converges and the last term involving $k_{s}$ goes to 0 as $k_{s} \rightarrow \infty$. So, for any $\varepsilon>0, k_{0}$ can be chosen to be suitably large to ensure that $R_{n}\left(k_{0}\right)<\varepsilon$, as desired.

We have a class of rules that depends on the value of $c$. Since we are free to choose any $c$, the issue is to find the value of $c$ that minimizes $\mathrm{E}(c)$ in (2.12). The following theorem shows that this minimum exists and is unique.

Theorem 2.3. $\mathrm{E}(c)=\frac{1}{2} \sum_{i=1}^{\infty} \alpha_{i-1} \beta_{i}$ is a strictly convex function of $c$.

Proof. Let $g_{i}=\log \left(\alpha_{i-1} \beta_{i}\right)$. Then, $\mathrm{E}(c)=\frac{1}{2} \sum_{i=1}^{\infty} \mathrm{e}^{g_{i}}, \mathrm{E}^{\prime}(c)=\frac{1}{2} \sum_{i=1}^{\infty} \mathrm{e}^{g_{i}} g_{i}^{\prime}$, and $\mathrm{E}^{\prime \prime}(c)=\frac{1}{2} \sum_{i=1}^{\infty} \mathrm{e}^{g_{i}}\left[\left(g_{i}^{\prime}\right)^{2}+g_{i}^{\prime \prime}\right]$.

It is therefore sufficient to show that $\left(g_{i}^{\prime}\right)^{2}+g_{i}^{\prime \prime} \geq 0$ for all $i$ with at least one inequality strict. It is straightforward to show that it is true for $i=1$ since $g_{1}=\log (c+1)$. For $i>1$,

$$
g_{i}=i \log (c+i)-i \log i+\sum_{j=1}^{i-1} \log j-\sum_{j=1}^{i-1} \log (c+j) .
$$

Hence,

$$
g_{i}^{\prime}=\frac{i}{c+i}-\sum_{j=1}^{i-1} \frac{1}{c+j}=\frac{1-\sum_{j=1}^{i-1}\left(h_{i, j}-1\right)}{c+i}
$$


where $h_{i, j}=(c+i) /(c+j)>1$ for $1 \leq j \leq i-1$. Similarly,

$$
g_{i}^{\prime \prime}=\frac{-i}{(c+i)^{2}}+\sum_{j=1}^{i-1} \frac{1}{(c+j)^{2}}=\frac{-1+\sum_{j=1}^{i-1}\left(h_{i, j}^{2}-1\right)}{(c+i)^{2}} .
$$

Hence,

$$
\begin{aligned}
(c+i)^{2}\left(\left(g_{i}^{\prime}\right)^{2}+g_{i}^{\prime \prime}\right) & =\left(1-\sum_{j=1}^{i-1}\left(h_{i, j}-1\right)\right)^{2}-1+\sum_{j=1}^{i-1}\left(h_{i, j}-1\right)\left(h_{i, j}+1\right) \\
& =1-2 \sum_{j=1}^{i-1}\left(h_{i, j}-1\right)+\left(\sum_{j=1}^{i-1}\left(h_{i, j}-1\right)\right)^{2}-1+\sum_{j=1}^{i-1}\left(h_{i, j}-1\right)\left(h_{i, j}+1\right) \\
& >0 .
\end{aligned}
$$

The inequality holds because $h_{i, j}+1>2$.

In Section 5 we list some values of the expected rank of the optimal rule, and compare them with the corresponding values of our simple rule, both when the latter stops with probability 1 , and with a given probability less than 1 . We find, numerically, the optimal $c$ values, which when stopping with probability 1 is about 2.4 and is not very sensitive to $n$, as seen in Tables 1 and 2 in Section 5.

\section{Retaining more than one item}

In this section we consider the performance of the simple rules given by (2.3), where we allow the rules to continue until a given number of items, $r$, are retained. To this end, we extend (2.8) for $\mathrm{P}\left(k_{s}, c\right)$, which provides the probability that at least one item is retained when $n \rightarrow \infty$, to the entire distribution for the number of items that these rules retain, asymptotically in $n$. Equation (2.11), which provides the expected absolute rank for the item that is retained by this rule is extended to the corresponding value when $r>1$. Finally, a discussion of the implementation of the optimal dynamic rule and its behavior (in terms of the relative rank that is required for retention as a function of the item number) for fixed $n$ is provided.

Let $Z_{n}$ denote the number of items that could be retained using the simple rule. Clearly, $Z_{n}=\sum_{i=1}^{n} I_{i}$, where $I_{1}, \ldots, I_{n}$ is a sequence of independent 0,1 random variables with $\mathrm{P}\left(I_{i}=\right.$ $1)=p_{\text {in }}=\min \left(\lfloor i c /(n+1-i)\rfloor, k_{s}\right) / i$, where $\lfloor x\rfloor$ is the largest integer less than or equal to $x$. Then $Z_{n}$ converges uniformly to a Poisson random variable (cf. [1, p. 3]). Let $p_{(n)}=$ $\max p_{i n}, i=1, \ldots, n$. Then

$$
\mathrm{P}\left(Z_{n}=r\right)=\frac{\mathrm{e}^{-\lambda_{n}} \lambda_{n}^{r}}{r !} \exp \left[O\left(\lambda_{n} p_{(n)}, r^{2} \lambda_{n}^{-1} p_{(n)}\right)\right]
$$

where $\lambda_{n}=\sum_{i=1}^{n} p_{\text {in }}$.

It remains to find the parameter $\lambda^{*}$ of the limiting Poisson distribution as $n \rightarrow \infty$. Following arguments similar to those in the previous section,

$$
\lambda^{*}=\lim _{n \rightarrow \infty} \sum_{k=1}^{k_{s}} \sum_{i=a_{n}(k)}^{b_{n}(k)} \frac{k}{i}=-\sum_{k=1}^{k_{s}} k \log f(k)=-\sum_{k=1}^{k_{s}} \log \left(\frac{k}{k+c}\right) .
$$

Specifically, Proposition 3.1, below, follows from [1]. 
Proposition 3.1. We have

$$
\lim _{n \rightarrow \infty} \mathrm{P}\left(Z_{n}=r\right)=\mathrm{e}^{-\lambda^{*}} \frac{\lambda^{* r}}{r !}
$$

The probability of retaining one item as $n \rightarrow \infty$ is $1-\lim _{n \rightarrow \infty} \mathrm{P}\left(Z_{n}=0\right)=1-\mathrm{e}^{-\lambda^{*}}$, which agrees with (2.8).

We now turn to the limiting expected sum of the absolute ranks, denoted by $\mathrm{E}_{2}\left(k_{s}, c\right)$, for keeping $r=2$ items, conditional on two items being kept, for simple rules given by (2.3) and fixed $k_{s}$ as $n \rightarrow \infty$. Let $A_{2}\left(k_{s}, c\right)$ be the limiting expected absolute rank of the second item that is kept, assuming that the first two available items (by the rule) are kept. Then the expected absolute sum of ranks from the two items kept (conditional on two items being kept) is

$$
\mathrm{E}_{2}\left(k_{s}, c\right)=\frac{\bar{F}(0) \mathrm{E}\left(k_{s}, c\right)+A_{2}\left(k_{s}, c\right)}{\bar{F}(1)},
$$

where $\bar{F}(x)=1-F(x)$ and $F$ is the cumulative distribution function of a Poisson random variable with parameter $\lambda^{*}$.

The expected absolute rank from the second item that is kept for fixed $n$ can be written in a form similar to (2.13) by summing over instances that item $k$ is the first item that is retained. Similar limiting arguments as $n \rightarrow \infty$ yield

$$
\begin{aligned}
A_{2}\left(k_{s}, c\right)=\sum_{k=1}^{k_{s}} V(k)( & {\left[\frac{k^{2}}{2(k+1)}\left(\frac{c+k}{k}-f(k) \frac{c+k+1}{k+1}\right)\right] } \\
& \left.+f(k) \frac{k}{2} \frac{c+k+1}{k+1} \log f(k)\right) \\
& -\sum_{k=1}^{k_{s}-1} \log f(k) \sum_{j=k+1}^{k_{s}} V(j) \frac{j}{2}\left(\frac{c+j}{j}-f(j) \frac{c+j+1}{j+1}\right) .
\end{aligned}
$$

Substituting (2.16) and (3.2) into (3.1) yields the desired result.

The rule can be modified so that the value of $c$ until the first item is retained, $c_{1}$, is different from the value of $c$ for selecting the second item, $c_{2}$. The numerical results of Table 5 in Section 5 show that, for the optimal choice of these $c$ values, $c_{1}>c_{2}$. This makes sense as this implies that the first item will be chosen rather early to allow for a sufficient number of items from which to make the second choice. This extends to retaining more than two items by means of different $c$ values.

In order to obtain the optimal rule which selects $r$ items by dynamic programming in an $n$-horizon problem, $n \geq r$, let $(m, j)$ be the state that the relative rank of item $m$ is being observed and $j$ additional items are required. Let $C(m, j)$ be the minimum over all stopping rules of the sum of the expected absolute ranks from the last $j$ items that are retained given that the state is $(m, j)$. The optimal solution to retain $r$ is then $C(1, r)$. This implies that the optimal solution for retaining $r$ out of $n, r=1, \ldots, n$, is obtained by one run of the dynamic program. Let $h(m, j)$ be the largest relative rank of an item to be retained in the optimal solution given that the state is $(m, j)$.

The values of $C(m, j)$ and $h(m, j)$ are determined recursively as follows:

$$
h(n, 1)=n \quad \text { and } \quad C(n, 1)=\frac{n+1}{2}, \quad C(n, j)=\infty \quad \text { for } j>1 .
$$


Furthermore, since it is preferred to retain item $m$ with $R_{m}=k$ in state $(m, j)$ if

$$
C(m+1, j-1)+\frac{(n+1) k}{(m+1)} \leq C(m+1, j),
$$

it follows that

$$
h(m, j)=\left\lfloor\frac{(C(m+1, j)-C(m+1, j-1))(m+1)}{n+1}\right\rfloor .
$$

This implies that

$$
\begin{aligned}
C(m, j)= & \frac{h(m, j)}{m}\left(C(m+1, j-1)+\frac{(n+1)(h(m, j)+1)}{2(m+1)}\right) \\
& +\left(1-\frac{h(m, j)}{m}\right) C(m+1, j) .
\end{aligned}
$$

Some values of $C(1, r)$ for various values of $n$ are given in Table 4 in Section 5.

\section{Rules that retain a given percentage of items}

In this section we consider simple rules which, for a fixed given fraction $\alpha, 0<\alpha<1$, retain (approximately) the fraction $\alpha$ of the population of 'good' items. More specifically, if, for fixed $\alpha$, we denote by $L_{n}(\alpha)$ the number of items retained by the rules considered, we show that

$$
\lim _{n \rightarrow \infty} \frac{\mathrm{E} L_{n}(\alpha)}{n}=\alpha
$$

and

$$
\frac{L_{n}(\alpha)}{n} \rightarrow \alpha \quad \text { a.s. as } n \rightarrow \infty .
$$

Let $S_{n}(\alpha)$ denote the sum of the absolute ranks of the items retained by the rule. Furthermore, we show that

$$
\lim _{n \rightarrow \infty} \mathrm{E} \frac{S_{n}(\alpha)}{n \mathrm{E}\left(L_{n}\right)}=\frac{\alpha}{2} .
$$

Note that this is optimal in the sense that if we could inspect all the items first, and pick the $\lceil\alpha n\rceil$ best items, then the sum of the ranks, $B_{n}$, would satisfy

$$
\lim _{n \rightarrow \infty} \frac{B_{n}}{n\lceil\alpha n\rceil}=\lim _{n \rightarrow \infty} \frac{\lceil\alpha n\rceil+1}{2 n}=\frac{\alpha}{2} .
$$

Let $0<\alpha<1$ be fixed. We omit the index $\alpha$. Consider the following rule.

Rule 4.1. Keep item $j$ if and only if $R_{j} \leq\lceil\alpha j\rceil$.

Note that knowledge of $n$ is not required to implement the rule. Let $I_{j}=1$ or 0 , according to whether or not the $j$ th item is kept. Then $L_{n}=\sum_{j=1}^{n} I_{j}$, and $\alpha \leq \mathrm{E} I_{j}<\alpha+1 / j, j=$ $1, \ldots, n$. Thus,

$$
n \alpha \leq \mathrm{E} L_{n}<\alpha n+\log n+1,
$$

and (4.1) follows. The second limit, (4.2), follows by noting that, by the independence of the $R_{j} \mathrm{~s}$, the $I_{j} \mathrm{~s}$ are independent (almost identically distributed) Bernoulli variables, and by applying the strong law of large numbers. 
Here $S_{n}=\sum_{j=1}^{n} A_{j}^{n} I_{j}$; thus,

$$
\begin{aligned}
\mathrm{E} S_{n} & =\sum_{j=1}^{n} \mathrm{E}\left(A_{j}^{n} I_{j}\right) \\
& =\sum_{j=1}^{n} \mathrm{E}\left(\mathrm{E} A_{j}^{n} I_{j} \mid R_{j}\right) \\
& =(n+1) \sum_{j=1}^{n} \frac{1}{j(j+1)} \sum_{k=1}^{\lceil j \alpha\rceil} k \\
& =\frac{n+1}{2} \sum_{j=1}^{n} \frac{\lceil j \alpha\rceil(\lceil j \alpha\rceil+1)}{j(j+1)} \\
& =\frac{n+1}{2}\left(n \alpha^{2}+o(n)\right) .
\end{aligned}
$$

Combining (4.4) and (4.5) yields (4.3).

We now show that the sum of the absolute ranks, $S_{n}$, suitably normalized, converges a.s. We first prove a result that shows that the normalized sum of the relative ranks of kept items converges a.s. to half of the sum of the resulting absolute ranks.

Lemma 4.1. Let $T_{n}=\sum_{i=1}^{n} R_{i} I_{i}$ (i.e. $T_{n}$ is the sum of the relative ranks from all items kept at the times that they are kept). Then

$$
\frac{T_{n}}{n L_{n}} \rightarrow \frac{\alpha}{4} \quad \text { a.s. as } n \rightarrow \infty .
$$

Proof. Let

$$
U_{i}=R_{i} I_{i}-\frac{v_{i}\left(v_{i}+1\right)}{2 i},
$$

where $v_{i}=\lceil\alpha i\rceil$. Then $U_{i}$ are independent random variables with mean zero. Let $b_{i}=i^{2}$. Since

$$
\sum_{i=1}^{\infty} \mathrm{E}\left(\frac{U_{i}^{2}}{b_{i}^{2}}\right)<\sum_{i=1}^{\infty} \frac{v_{i}\left(v_{i}+1\right)\left(2 v_{i}+1\right)}{6 i^{5}}<\infty,
$$

it follows from [3, Theorem 2, p. 239] that $b_{n}^{-1} \sum_{i=1}^{n} U_{i} \rightarrow 0$ a.s. as $n \rightarrow \infty$. This implies that $T_{n} / n^{2} \rightarrow \alpha^{2} / 4$ a.s. because $n^{-2} \sum_{i=1}^{n}\left[v_{i}\left(v_{i}+1\right)\right] /(2 i) \rightarrow \alpha^{2} / 4$. Equation (4.6) follows from (4.2).

We are now prepared to show the following theorem.

Theorem 4.1. We have

$$
\frac{S_{n}}{n L_{n}} \rightarrow \frac{\alpha}{2} \quad \text { a.s. as } n \rightarrow \infty .
$$

Proof. Let $G_{n}$ be the sum over $j=1, \ldots, n$ of the contribution to the absolute rank $A_{j}^{n}$ above $R_{j}$ for the items retained, i.e. $G_{n}=\sum_{j=1}^{n}\left(A_{j}^{n}-R_{j}\right) I_{j}$. Then, by (2.9),

$$
\mathrm{E}\left(G_{n} \mid \mathcal{F}_{n-1}\right)=\left(G_{n-1}+R_{n-1} I_{n-1}\right) \frac{(n+1)}{n} .
$$


Let $Z_{n}=G_{n} / n(n+1)$. Equation (4.7) implies that

$$
\mathrm{E}\left(Z_{n} \mid \mathcal{F}_{n-1}\right)=\frac{Z_{n-1}(n-1) n(n+1)}{n(n+1) n}+\frac{R_{n-1} I_{n-1}}{n^{2}}=\frac{n-1}{n} Z_{n-1}+\frac{R_{n-1} I_{n-1}}{n^{2}} .
$$

Note that, by Lemma $4.1, \sum_{n=2}^{\infty} I_{n-1} R_{n-1} / n^{2} \rightarrow \alpha^{2} / 4$ a.s. Hence, $G_{n} / n^{2}$ a.s. converges by Theorem 1 of [6]. But, $T_{n} / n^{2}$ converges a.s. by Lemma 4.1 and $S_{n} / n^{2}=\left(G_{n}+T_{n}\right) / n^{2}$. Therefore, $S_{n} / n^{2}$ converges a.s.

To see that the almost-sure limit of $S_{n} / n^{2}$ is $\alpha^{2} / 2$, note that $S_{n} \geq L_{n}\left(L_{n}+1\right) / 2$ and, thus, by (4.2), $\lim \inf S_{n} / n^{2} \geq \alpha^{2} / 2$ a.s. Since $S_{n} / n^{2}$ is bounded, it follows that

$$
\mathrm{E}\left(\lim \frac{S_{n}}{n^{2}}\right)=\lim \mathrm{E}\left(\frac{S_{n}}{n^{2}}\right)=\frac{\alpha^{2}}{2}
$$

where the last equality follows from (4.1) and (4.3). Thus, if $\mathrm{P}\left(\lim S_{n} / n^{2}>\alpha^{2} / 2\right)>0$, we would get a contradiction.

The simple rule discussed above satisfies $L_{n} /\lceil\alpha n\rceil \rightarrow 1$ a.s. Suppose, however, that it is desired that a rule keep exactly $\lceil\alpha n\rceil$ items. Can this be achieved? Below we exhibit a rule for which $\mathrm{P}\left(L_{n}=\lceil\alpha n\rceil\right) \rightarrow 1$ and for which (4.3) holds. This is achieved by increasing the cutoff level for which an item is kept by a small amount.

Rule 4.2. Fix $0<\alpha<1$ and $0<\omega<0.5$. Retain item $j$ if

$$
R_{j} \leq\left\lceil\left(\alpha+n^{-\omega}\right) j\right\rceil
$$

and if $\lceil\alpha n\rceil$ items have not already been chosen.

Proposition 4.1. For Rule 4.2,

$$
\mathrm{P}\left(L_{n}=\lceil\alpha n\rceil\right) \rightarrow 1 \text { as } n \rightarrow \infty .
$$

Proof. Let $X_{n}$ be the number of items $j$ for which (4.8) holds, $j=1, \ldots, n$. We shall show that $\mathrm{P}\left(X_{n} \geq\lceil\alpha n\rceil\right) \rightarrow 1$. Let $I_{j}=1$ if (4.8) holds for $j$ and $I_{j}=0$ otherwise. Then $\mathrm{P}\left(I_{j}=1\right)=p_{n j} \geq p_{n}=\alpha+n^{-\omega}$. Let $Y_{n}$ be a $\operatorname{binomial}\left(n, p_{n}\right)$ random variable. Then $X_{n}$ is stochastically larger than or equal to $Y_{n}$. Hence,

$$
\begin{aligned}
\lim _{n \rightarrow \infty} \mathrm{P}\left(X_{n} \geq\lceil\alpha n\rceil\right) & \geq \lim _{n \rightarrow \infty} \mathrm{P}\left(Y_{n} \geq\lceil\alpha n\rceil\right) \\
& =\lim _{n \rightarrow \infty}\left(1-\Phi\left(\frac{\lceil\alpha n\rceil-n p_{n}}{\sqrt{n p_{n}\left(1-p_{n}\right)}}\right)\right) \\
& \geq \lim _{n \rightarrow \infty}\left(1-\Phi\left(\frac{1-n^{1-\omega}}{\sqrt{n p_{n}\left(1-p_{n}\right)}}\right)\right) \\
& =1,
\end{aligned}
$$

since $0<\omega<0.5$, where $\Phi$ is the standard normal distribution. Thus, (4.9) holds.

Proposition 4.2. Equation (4.3) holds for Rule 4.2.

Proof. We have

$$
\mathrm{E}\left(A_{j}^{n} \mid I_{j}=1\right) \leq \frac{n+1}{j+1}\left(\frac{\left(\alpha+n^{-\omega}\right) j+1}{2}\right) \leq(n+1)\left(\frac{\alpha+n^{-\omega}}{2}+\frac{1}{2(j+1)}\right) .
$$


Let $j_{1}^{*}, \ldots, j_{s}^{*}$ be the at most $\lceil\alpha n\rceil$ smallest indices $j \leq n$ for which (4.8) holds. Thus, $S_{n}=\sum_{i=1}^{n} A_{j_{i}^{*}}^{n} I_{j_{i}^{*}}$. Hence,

$$
\mathrm{E} S_{n} \leq \frac{\lceil\alpha n\rceil(n+1)\left(\alpha+n^{-\omega}\right)}{2}+\frac{(n+1) \log (n+2)}{2} .
$$

It follows that $\mathrm{E} S_{n} /\lceil\alpha n\rceil n \rightarrow \alpha / 2$ as $n \rightarrow \infty$, i.e. (4.3) holds.

A result corresponding to Theorem 4.1, for Rule 4.2, follows in a similar way as for Rule 4.1.

\section{Numerical results}

In this section we demonstrate the results from the previous sections. We first illustrate the findings from Section 2 where one item is retained. Table 1 presents the expected value of the absolute rank of the retained item of the optimal rule (based on dynamic programming for each given $n$ ), and the corresponding value and the optimal choice of $c$ for the simple rule, which is evaluated directly for each given $c$.

It is apparent from Table 1 that the relative advantage of using the simple rule is greater for large $n$. It is interesting that the performance of the simple rule is less sensitive to the value of $n$. Hence, when the number of items becomes large, the case where it is hard to implement dynamic programming, is when the simple rule performs almost as well as the optimal rule.

The choice of $c$ in the simple rule should be taken to be about 2.4. This value of $c$ is nearly optimal for a wide range of $n$. Furthermore, the actual performance does not vary much for different $c$ near 2.4, as seen in Table 2. Note from Table 2 that, for $n=100, c=2.30$ performs better than $c=2.4$, consistent with the findings in Table 1 .

In Table 3 we illustrate the performance of the rules which select an item with probability $\mathrm{P}<1$, along with their optimal $c$ and $k_{s}$. It is observed that even for $\mathrm{P}=0.99$ we do not need

TABLE 1: Optimal and simple rules for retaining one item.

\begin{tabular}{rccc}
\hline \multicolumn{1}{c}{$n$} & $\begin{array}{c}\text { Optimal } \\
\text { expected rank }\end{array}$ & $\begin{array}{c}\text { Simple rule } \\
\text { expected rank }\end{array}$ & Optimal $c$ \\
\hline 100 & 3.6032 & 3.7348 & 2.30 \\
1000 & 3.8324 & 3.9062 & 2.36 \\
10000 & 3.8649 & 3.9258 & 2.41 \\
100000 & 3.8690 & 3.9279 & 2.41 \\
1000000 & 3.8695 & 3.9281 & 2.42 \\
\hline
\end{tabular}

TABLE 2: Sensitivity of the one-choice simple rule to $c$.

\begin{tabular}{cccccc}
\hline \multirow{2}{*}{$c$} & \multicolumn{5}{c}{$n$} \\
\cline { 2 - 6 } & 100 & 1000 & 10000 & 100000 & 1000000 \\
\hline 2.00 & 3.7706 & 4.0019 & 4.0397 & 4.0450 & 4.0457 \\
2.10 & 3.7550 & 3.9543 & 3.9857 & 3.9894 & 3.9899 \\
2.20 & 3.7418 & 3.9251 & 3.9513 & 3.9543 & 3.9546 \\
2.30 & 3.7348 & 3.9109 & 3.9325 & 3.9349 & 3.9352 \\
2.40 & 3.7405 & 3.9066 & 3.9259 & 3.9280 & 3.9282 \\
2.50 & 3.7540 & 3.9111 & 3.9292 & 3.9311 & 3.9313 \\
\hline
\end{tabular}


TABLE 3: One item selected with given $\mathrm{P}$, optimal $c$ and $k_{s}$, conditional expected absolute rank, and the actual value of $\mathrm{P}$ obtained.

\begin{tabular}{|c|c|c|c|c|}
\hline $\mathrm{P}$ & $\begin{array}{c}\text { Actual } \\
\text { probability }\end{array}$ & $c$ & $k_{s}$ & $\begin{array}{c}\text { Expected absolute } \\
\text { rank }\end{array}$ \\
\hline \multicolumn{5}{|c|}{$n=100$} \\
\hline 0.50 & 0.5051 & 1.06 & 1 & 1.5049 \\
\hline 0.60 & 0.6061 & 1.58 & 1 & 1.7549 \\
\hline 0.70 & 0.7013 & 1.15 & 2 & 1.8982 \\
\hline 0.80 & 0.8032 & 1.25 & 3 & 2.2058 \\
\hline 0.90 & 0.9013 & 1.61 & 4 & 2.6028 \\
\hline 0.95 & 0.9506 & 1.74 & 6 & 2.9378 \\
\hline 0.99 & 0.9906 & 2.16 & 11 & 3.4140 \\
\hline \multicolumn{5}{|c|}{$n=1000$} \\
\hline 0.50 & 0.5015 & 1.01 & 1 & 1.5025 \\
\hline 0.60 & 0.6016 & 1.51 & 1 & 1.7536 \\
\hline 0.70 & 0.7021 & 1.14 & 2 & 1.9176 \\
\hline 0.80 & 0.8010 & 1.22 & 3 & 2.2254 \\
\hline 0.90 & 0.9001 & 1.60 & 4 & 2.6346 \\
\hline 0.95 & 0.9502 & 1.96 & 5 & 2.9817 \\
\hline 0.99 & 0.9901 & 2.15 & 11 & 3.4964 \\
\hline \multicolumn{5}{|c|}{$n=10000$} \\
\hline 0.50 & 0.5025 & 1.01 & 1 & 1.5049 \\
\hline 0.60 & 0.6016 & 1.51 & 1 & 1.7548 \\
\hline 0.70 & 0.7023 & 1.14 & 2 & 1.9193 \\
\hline 0.80 & 0.8011 & 1.22 & 3 & 2.2277 \\
\hline 0.90 & 0.9004 & 1.60 & 4 & 2.6391 \\
\hline 0.95 & 0.9502 & 1.96 & 5 & 2.9868 \\
\hline 0.99 & 0.9900 & 2.15 & 11 & 3.5035 \\
\hline \multicolumn{5}{|c|}{$n=100000$} \\
\hline 0.50 & 0.5025 & 1.01 & 1 & 1.5050 \\
\hline 0.60 & 0.6016 & 1.51 & 1 & 1.7550 \\
\hline 0.70 & 0.7000 & 1.13 & 2 & 1.9142 \\
\hline 0.80 & 0.8011 & 1.22 & 3 & 2.2279 \\
\hline 0.90 & 0.9005 & 1.60 & 4 & 2.6395 \\
\hline 0.95 & 0.9502 & 1.96 & 5 & 2.9873 \\
\hline 0.99 & 0.9900 & 2.15 & 11 & 3.5043 \\
\hline \multicolumn{5}{|c|}{$n=1000000$} \\
\hline 0.50 & 0.5025 & 1.01 & 1 & 1.5050 \\
\hline 0.60 & 0.6016 & 1.51 & 1 & 1.7550 \\
\hline 0.70 & 0.7000 & 1.13 & 2 & 1.9142 \\
\hline 0.80 & 0.8011 & 1.22 & 3 & 2.2280 \\
\hline 0.90 & 0.9005 & 1.60 & 4 & 2.6396 \\
\hline 0.95 & 0.9502 & 1.96 & 5 & 2.9874 \\
\hline 0.99 & 0.9900 & 2.15 & 11 & 3.5044 \\
\hline
\end{tabular}


TABLE 4: Expected absolute rank/( $(r+1) / 2)$ obtained by the optimal rule for keeping $r$.

\begin{tabular}{rccccc}
\hline$r$ & \multicolumn{5}{c}{$n$} \\
\cline { 2 - 6 } & 100 & 1000 & 10000 & 100000 & 1000000 \\
\hline 1 & 3.6032 & 3.8324 & 3.8649 & 3.8690 & 3.8695 \\
2 & 2.7937 & 2.9781 & 3.0039 & 3.0072 & 3.0076 \\
3 & 2.3774 & 2.5386 & 2.5612 & 2.5640 & 2.5644 \\
4 & 2.1216 & 2.2688 & 2.2893 & 2.2919 & 2.2922 \\
5 & 1.9476 & 2.0851 & 2.1042 & 2.1066 & 2.1069 \\
6 & 1.8209 & 1.9513 & 1.9694 & 1.9716 & 1.9719 \\
7 & 1.7242 & 1.8491 & 1.8664 & 1.8686 & 1.8688 \\
8 & 1.6475 & 1.7683 & 1.7850 & 1.7871 & 1.7873 \\
9 & 1.5849 & 1.7026 & 1.7188 & 1.7208 & 1.7211 \\
10 & 1.5331 & 1.6481 & 1.6639 & 1.6659 & 1.6661 \\
\hline
\end{tabular}

TABLE 5: Two items selected with probability 1 for equal $c$ and unequal $c_{1}$ and $c_{2}$, optimal $c, c_{1}$, and $c_{2}$, and the expected absolute rank (EAR)/3.

\begin{tabular}{rcccccc}
\hline \multicolumn{1}{c}{$n$} & $c$ & $c_{1}$ & $c_{2}$ & $\begin{array}{r}\mathrm{EAR} / 3 \\
\text { (equal) }\end{array}$ & $\begin{array}{c}\mathrm{EAR} / 3 \\
\text { (unequal) }\end{array}$ & $\begin{array}{c}\mathrm{EAR} / 3 \\
\text { (optimal) }\end{array}$ \\
\hline 100 & 3.21 & 3.81 & 2.16 & 3.0857 & 2.8667 & 2.7937 \\
1000 & 3.19 & 3.79 & 2.20 & 3.2198 & 3.0078 & 2.9798 \\
10000 & 3.15 & 3.80 & 2.22 & 3.2342 & 3.0259 & 3.0039 \\
\hline
\end{tabular}

$k_{s}$ bigger than 11 , for all $n \leq 10^{6}$. It is noted that, for $n \geq 1000$, the optimal values of $c$ and $k_{s}$ do not change with $n$ (up to the precision given).

Table 4 lists the optimal sum of the ranks for keeping $r$, standardized by $r(r+1) / 2$ (which is the lowest possible attainable sum of $r$ ranks). These values were obtained by the dynamic programming described in Section 3.

In Table 5 we list the performance of the simple rule which selects two items, with one $c$ value and with $c_{1} \neq c_{2}$, respectively. It is seen that $c_{1} \neq c_{2}$ yields a large improvement. The optimal values are listed for comparison.

In Table 6 we list the corresponding conditional values when a selection is only required with given probability $\mathrm{P}$. The same $c$ is used for both choices. A comparison with the corresponding values in Table 3 shows that the second term improves the average (when divided by $r(r+1) / 2=3)$.

Finally, in Table 7 we show the performance of Rule 4.1 for $\alpha=0.1,0.2,0.3,0.4$, and 0.5 and $n=100,1000,10000$. This is the only table that is based on simulations, with 1000 replications for each $n$ and $\alpha$. Since the probability of retaining an item is usually greater than $\alpha$ and at least equal to $\alpha$, it is not surprising that the average number kept, when standardized by $n \alpha$, is somewhat greater than 1 . This effect is larger for small values of $n$. Similarly, the average absolute rank standardized by $n / 2$ times the number kept, is slightly above $\alpha$, especially when $n$ is small. 
TABLE 6: Two items selected with given $\mathrm{P}$, optimal $k_{s}$, and the conditional expected absolute rank (EAR)/3.

\begin{tabular}{|c|c|c|c|c|c|}
\hline$n$ & $\mathrm{P}$ & $\begin{array}{c}\text { Actual } \\
\text { probability }\end{array}$ & $c$ & $k_{s}$ & $\mathrm{EAR} / 3$ \\
\hline \multirow[t]{7}{*}{100} & 0.50 & 0.5365 & 2.04 & 2 & 1.9407 \\
\hline & 0.60 & 0.6030 & 2.48 & 2 & 2.0179 \\
\hline & 0.70 & 0.7037 & 2.20 & 3 & 2.0724 \\
\hline & 0.80 & 0.8024 & 2.31 & 4 & 2.2093 \\
\hline & 0.90 & 0.9007 & 2.42 & 6 & 2.4414 \\
\hline & 0.95 & 0.9502 & 2.62 & 8 & 2.6107 \\
\hline & 0.99 & 0.9903 & 3.06 & 13 & 2.8939 \\
\hline \multirow[t]{7}{*}{1000} & 0.50 & 0.5019 & 1.82 & 2 & 1.9436 \\
\hline & 0.60 & 0.6003 & 1.66 & 3 & 2.0209 \\
\hline & 0.70 & 0.7009 & 2.18 & 3 & 2.1091 \\
\hline & 0.80 & 0.8013 & 2.32 & 4 & 2.2568 \\
\hline & 0.90 & 0.9004 & 2.48 & 6 & 2.4940 \\
\hline & 0.95 & 0.9503 & 2.70 & 8 & 2.6919 \\
\hline & 0.99 & 0.9901 & 2.95 & 15 & 2.9935 \\
\hline \multirow[t]{7}{*}{10000} & 0.50 & 0.5016 & 1.82 & 2 & 1.9468 \\
\hline & 0.60 & 0.6002 & 1.66 & 3 & 2.0235 \\
\hline & 0.70 & 0.7002 & 2.18 & 3 & 2.1119 \\
\hline & 0.80 & 0.8009 & 2.32 & 4 & 2.2607 \\
\hline & 0.90 & 0.9007 & 2.49 & 6 & 2.5011 \\
\hline & 0.95 & 0.9504 & 2.71 & 8 & 2.7002 \\
\hline & 0.99 & 0.9902 & 2.96 & 15 & 3.0023 \\
\hline
\end{tabular}

TABLE 7: The average absolute rank normalized by [the number of items kept times $n / 2$ ], (A), the average number of items kept normalized by $n \alpha$, (B), and their standard deviations for Rule 4.1 and selecting a proportion $\alpha$.

\begin{tabular}{rccccc}
\hline \multicolumn{1}{c}{$n$} & $\alpha$ & $(\mathrm{A})$ & Standard deviation of $(\mathrm{A})$ & $(\mathrm{B})$ & Standard deviation of $(\mathrm{B})$ \\
\hline 100 & 0.1 & 0.1760 & 0.06500 & 1.2291 & 0.3305 \\
100 & 0.2 & 0.2579 & 0.05604 & 1.1114 & 0.2101 \\
100 & 0.3 & 0.3457 & 0.05306 & 1.0582 & 0.1621 \\
100 & 0.4 & 0.4457 & 0.05276 & 1.0469 & 0.1253 \\
100 & 0.5 & 0.5572 & 0.05041 & 1.0572 & 0.0967 \\
1000 & 0.1 & 0.1127 & 0.01351 & 1.0386 & 0.0964 \\
1000 & 0.2 & 0.2092 & 0.01435 & 1.0171 & 0.0651 \\
1000 & 0.3 & 0.3078 & 0.01636 & 1.0102 & 0.0514 \\
1000 & 0.4 & 0.4080 & 0.01566 & 1.0095 & 0.0376 \\
1000 & 0.5 & 0.5091 & 0.01692 & 1.0098 & 0.0327 \\
10000 & 0.1 & 0.1017 & 0.00325 & 1.0055 & 0.0302 \\
10000 & 0.2 & 0.2013 & 0.00396 & 1.0025 & 0.0195 \\
10000 & 0.3 & 0.3012 & 0.00476 & 1.0019 & 0.0156 \\
10000 & 0.4 & 0.4012 & 0.00495 & 1.0015 & 0.0123 \\
10000 & 0.5 & 0.5012 & 0.00502 & 1.0014 & 0.0100 \\
\hline
\end{tabular}




\section{Concluding remarks}

The main thrust of the paper is to find simple rules that perform nearly optimally. A referee provided an alternative elegant approach to deriving the asymptotic results that appear in Sections 2 and 3. This derivation motivates extensions to other rules that are perhaps less simple.

Consider what happens in the limit as the number of items, $n$, goes to infinity. Assume that the times that the observations are observed are equally spaced on the interval $(0,1]$. Let $N_{i}(s, t)$ denote the number of items that have relative rank $i$ on the time interval $(s, t), 0<s<t \leq 1$. The referee observed that $N_{i}(s, t)$ has a Poisson distribution with mean

$$
m_{i}(s, t)=\int_{s}^{t} \frac{1}{x} \mathrm{~d} x=\log \left(\frac{t}{s}\right) .
$$

Hence, the distribution of $N_{i}(s, t)$ does not depend on $i$.

Using the above leads to an alternative approach for obtaining the asymptotic results that appear in Sections 2 and 3. For example, consider the curtailed rule that is defined by $0<t_{1} \leq$ $t_{2} \leq \cdots \leq t_{k_{s}} \leq 1$ for a given $k_{s}$. This rule chooses an item if it has a relative rank $i$, provided that it arrives after time $t_{i}$ and the number of items that are already retained is not equal to the given quota of $r$. If the objective is to retain one item then it is easily seen that the expected absolute rank conditional on retaining one item is

$$
\mathrm{E}\left(k_{s}\right)=\frac{\sum_{k=1}^{k_{s}} V(k) / t_{k}-k_{s}\left(1-\mathrm{P}\left(k_{s}\right)\right)}{2 \mathrm{P}\left(k_{s}\right)},
$$

where $V(1)=1, V(k)=t_{1} \cdots t_{k-1} / t_{k}^{k-1}$ for $1<k \leq k_{s}$, and the probability of stopping is $\mathrm{P}\left(k_{s}\right)=1-t_{1} t_{2} \cdots t_{k_{s}}$.

The simple rule in Section 2 is of the form $t_{k}=k /(k+c), 1 \leq k \leq k_{s}$. To illustrate an extension based on the observation of the referee, assume that $k_{s}=2$ and consider minimizing $\mathrm{E}\left(k_{s}\right)$ subject to a probability of $p$ that one item is not retained. The optimal choices of $t_{1}$ and $t_{2}$ are found by minimizing

$$
f\left(t_{1}, t_{2}\right)=\frac{1 / t_{1}+t_{1} / t_{2}^{2}-2 t_{1} t_{2}}{2\left(1-t_{1} t_{2}\right)}
$$

subject to $t_{1} t_{2}=p$. It is straightforward to find that the optimal values are $t_{1}=\sqrt{p} / d$ and $t_{2}=\sqrt{p} d$, where $d=3^{1 / 4}$ unless $\sqrt{p} d>1$, in which case $t_{2}=1$ and $t_{1}=p$. For the simple rule in Section 2, the value of $t_{k}=k /(k+c)$ for $k=1,2$. It is straightforward to find that $c=(\sqrt{1+8 / p}-3) / 2$ to achieve a probability of $p$ of not retaining two items.

If the probability that an item is not retained is set to be $10 \%$, that is, $p=0.1$, for example, then the optimal values of $t_{1}$ and $t_{2}$ are 0.240 and 0.416 , respectively, with $\mathrm{E}(2)=2.972$. In contrast, for the simple rule described in the paper, $c=3$. Hence, $t_{1}=0.25$ and $t_{2}=0.4$ with $\mathrm{E}(2)=2.979$. The optimization problem becomes more difficult when either $r>1$ or $k_{s}>2$. We only illustrate this idea and do not explore it further as the objective of this paper is to describe simple rules that perform well.

\section{Acknowledgements}

We would like to thank an anonymous referee for the wonderful insight that we incorporated in Section 6 and the careful reading of our paper. We also would like to thank Moshe Pollak for many helpful suggestions. 


\section{References}

[1] Barbour, A. D., Holst, L. And Janson, S. (1992). Poisson Approximation. Oxford University Press.

[2] Chow, Y. S., Moriguti, S., Robbins, H. and Samuels, S. M. (1964). Optimal selection based on relative rank (the 'secretary problem'). Israel J. Math 2, 81-90.

[3] Feller, W. (1971). An Introduction to Probability Theory and Its Applications, Vol. 2, 2nd edn. John Wiley, New York.

[4] Ferguson, T. S. (2002). Optimal Stopping and Applications. Available at http://www.math.ucla.edu/ tom/ stopping/contents.html.

[5] Krieger, A. M., Pollak, M. and Samuel-Cahn, E. (2007). Select sets: rank and file. Ann. Appl. Prob. 17, 360-385.

[6] Robiins, H. And Siegmund, D. (1971). A convergence theorem for nonnegative almost supermartingales and some applications. In Optimizing Methods in Statistics, ed. J. S. Rustagi, Academic Press, New York, pp. 233257.

[7] Yeo, A. J. And Yeo, G. F. (1994). Selecting satisfactory secretaries. Austral. J. Statist. 36, 185-198. 